\title{
The Use of I-gel laryngeal mask with selective endobronchial blocker for uniportal video assisted thoracic
} surgery

\author{
Guido Di Gregorio ${ }^{1}$, Nicolò Sella ${ }^{1}$, Alessandro Pangoni ${ }^{2}$, Demetrio Pittarello ${ }^{1}$, Paolo Navalesi ${ }^{1}$, Federico Rea ${ }^{2}$ and Andrea Dell'Amore ${ }^{2 *}$ \\ ${ }^{1}$ Department of Medicine, Anesthesiology and Intensive Care, University of Padova, Padova, Italy \\ ${ }^{2}$ Department of Cardiac, Thoracic, Vascular Sciences and Public Health, Thoracic Surgery Unit, University of Padova, Italy
}

\begin{abstract}
The development of video-assisted thoracoscopic surgery has led to a rising interest in decreasing the invasiveness not only of the surgical procedures, but also of the anaesthetic management. We report our preliminary experience using a supraglottic device and a bronchial blocker without neuromuscular blockade during uniportal video assisted thoracic surgery.
\end{abstract}

\section{Introduction}

General anaesthesia with curarization and tracheal intubation has always been considered mandatory for thoracic surgery to provide selective one lung ventilation in order to achieve an optimal exposure of the surgical field. However, the risks of this technique are well-described, in particular iatrogenic tracheobronchial injuries related to orotracheal intubation [1], the development of volutrauma or barotrauma as well as atelectasis [2], and postoperative residual neuromuscular blockade [3].

In the last years, the development of video-assisted thoracoscopic surgery (VATS) has led to a rising interest in decreasing the invasiveness not only of the surgical procedures, but also of the anaesthetic management, thus several authors investigated the role of nonintubated and awake thoracic surgery with various anaesthesiologic techniques [4].

We report three cases of non-intubated VATS providing one lung ventilation with I-gel laryngeal mask and Arndt endobronchial blocker without neuromuscular blockade.

\section{Case series}

\section{Case 1}

A 75 year old man (weight $70 \mathrm{~kg}$, height $161 \mathrm{~cm}$ ) was listed for elective uniportal thoracoscopic pleural biopsy. One year before he underwent a thoracoscopic left superior lobectomy for lung adenocarcinoma, but since a month he has been complaining of a symptomatic left pleural effusion, that was already drained without reaching a clear cytologic diagnosis. He had a history of ischemic cardiomyopathy with coronaryaortic bypass grafting one year before, ischemic stroke and type 2 diabetes mellitus. The preoperative pulmonary function test showed a restrictive-obstructive deficit (FEV1 48\%, FEV1/FVC 74\%).

In the operating theatre a large venous catheter was positioned in a peripheral vein and two-lead electrocardiogram, pulse-oximetry values and arterial blood pressure were recorded. An ultrasound-guided T5 erector spinae plane block was performed with Ropivacaine $0.5 \% 20$
$\mathrm{mL}$ single shot and then anaesthesia was inducted with Propofol $2 \mathrm{mg} /$ $\mathrm{kg}$ and Fentanyl $1.5 \mathrm{mcg} / \mathrm{kg}$. No neuromuscular blocking agent was administered. An I-gel 4 laryngeal mask was positioned (Figure 1A) and an Arndt endobronchial blocker was placed through the I-gel mask with fiberoptic bronchoscopy guidance into the left main bronchus (Figure 1B). Propofol $7 \mathrm{mg} / \mathrm{kg} / \mathrm{h}$ was administered for sedation during the surgery without other analgesic requirement and the patient was mechanically ventilated with $7 \mathrm{~mL} / \mathrm{kg}$ tidal volume.

The patient was positioned in a right lateral decubitus and the position of the endobronchial blocker was re-checked (Figure 1C). The surgical exposure was defined adequate by the surgeon (unaware of the anaesthetic technique) and a uniportal (Figure 1D) thoracoscopic multiple pleural biopsy procedure was performed uneventfully in 43 minutes. Peripheral oxygen saturation, end-tidal carbon dioxide and airways pressures have always been within the normal range.

When one lung ventilation was no longer required, the endobronchial blocker was deflated and positive pressure ventilation was delivered to the left lung. At the end of the surgery, when the patient met the standard criteria for extubation, the I-gel mask was removed en block with Arndt blocker and the patient was transferred back to the ward with $98 \%$ of peripheral oxygen saturation in fresh air. No pain, sore throat and hoarseness of voice were complained.

The postoperative stay was regular and the patient was discharged at home three days later.

${ }^{*}$ Correspondence to: Andrea Dell'Amore, Department of Cardiac, Thoracic, Vascular Sciences and Public Health, Thoracic Surgery Unit, University of Padova, Italy, Tel: +390498212242; E-mail: dellamore76@libero.it

Key words: I-gel laryngeal mask, video-assisted thoracoscopic surgery, supraglottic airway devices, non-intubated VATS

Received: December 02, 2020; Accepted: December 08, 2020; Published: December 11, 2020 


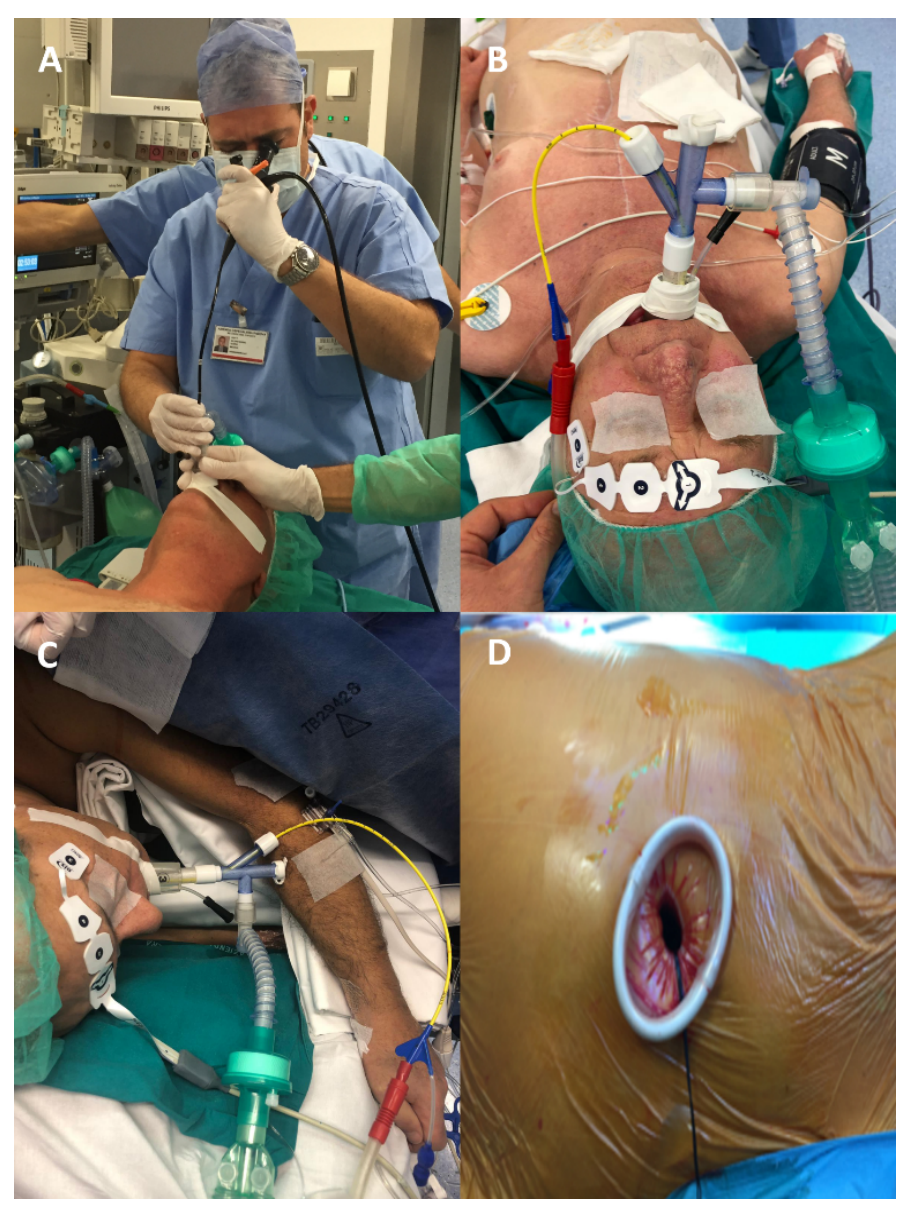

Figure 1. A) the correct position of the mask is checked with bronchoscope. B) the bronchial blocker is positioned under bronchoscope control. C) the patient is placed in lateral decubitus. D) intraoperative picture of the single access for video-assisted thoracic procedure

\section{Case 2}

A 62 year old man (weight $73 \mathrm{~kg}$, height $173 \mathrm{~cm}$ ) was scheduled for elective uniportal thoracoscopic resection of a PET positive mediastinal mass located at aortopulmonary window. His medical history comprises a stage IV B-cell non-Hodgkin lymphoma (treated with chemotherapy), arterial hypertension and a smoking attitude. The preoperative ECG, laboratory test and pulmonary function test were normal.

After standard monitoring with electrocardiogram, arterial blood pressure and pulse-oximetry, a peripheral venous catheter was positioned and an ultrasound-guided T5 erector spinae plane block was performed with Ropivacaine $0.5 \% 20 \mathrm{~mL}$ single shot. The patient was sedated with Propofol $3 \mathrm{mg} / \mathrm{kg}$ and Fentanyl $2 \mathrm{mcg} / \mathrm{kg}$ without neuromuscular blockade: the I-gel 4 laryngeal mask was positioned and an Arndt endobronchial blocker was placed through the I-gel mask with fiberoptic bronchoscopy guidance into the left main bronchus. The anaesthetic management was similar to the previous case. The surgeon was satisfied with the lung collapse and the procedure lasted 60 minutes without complications. Vital parameters were always stable and peak airways pressures were within normal ranges. Lung re-inflation was easy and adequate. The I-gel mask and the Arndt blocker were removed at the end of the surgery and the patient was transferred back to the ward with $100 \%$ peripheral oxygen saturation in oxygen mask $4 \mathrm{~L} /$ minute: the greatest postoperative pain was classified as NRS 2 and no sore throat and hoarseness of voice were complained. The patient was discharged at home three days later.

\section{Case 3}

A 65 year old man (weight $97 \mathrm{~kg}$, height $189 \mathrm{~cm}$ ) was planned for elective uniportal thoracoscopic biopsy of a large mediastinal mass $(14.5 \times 7.5 \times 6.5 \mathrm{~cm})$ with severe obstruction of the trachea and of the main bronchi, already treated with radiotherapy for dyspnoea. The patient had no previous medical history. Postoperative discharge to Intensive Care Unit was pre-set.

In the operating theater physiologic monitoring was undertaken, and two peripheral intra- venous cannulas and an arterial line were inserted. An ultrasound-guided T5+T7 erector spinae plane block was performed with Ropivacaine $0.5 \% 15 \mathrm{~mL}+15 \mathrm{~mL}$ single shot. After the induction of anaesthesia with Propofol $2 \mathrm{mg} / \mathrm{kg}$ and Fentanyl $2 \mathrm{mcg} / \mathrm{kg}$, an I-gel 5 laryngeal mask was positioned and an Arndt endobronchial blocker was placed through the I-gel mask with fiberoptic bronchoscopy guidance into the left main bronchus. The anaesthetic management was similar to the previous cases. Lung isolation was satisfactory and the surgical time was 55 minutes. Oxygen saturation, end-tidal carbon dioxide and airways pressures were always within the normal range.

At the end of the procedure the patient was extubated and transferred back to the ward without the need for Intensive Care Unit monitoring. Postoperative stay was complicated by subcutaneous emphysema and the patient was discharged at home four days later.

\section{Discussion}

General anaesthesia using double-lumen intubation with onelung ventilation is the most frequent anaesthesiologic techniques used for thoracic surgery procedures. Undoubted advantages of this technique include easy lung isolation, quick switch back and forth between one lung and two lung ventilation, and simple suctioning of both lungs. Nevertheless, general anaesthesia with tracheal intubation was associated with increased risk for pneumonia and damages to the lung parenchyma due to mechanical ventilation [2,5]. Moreover, several studies reported iatrogenic tracheobronchial injuries with high morbidity and mortality [1]. Finally, placement of a dual lumen tube is more complicated than placement of a single lumen tracheal tube, especially in patients with difficult airway, distorted lower airway, or tracheostomy.

For these reasons, in the past years different anaesthetic techniques for thoracic surgery were investigated in order to reduce the invasiveness of the anaesthetic approach and to avoid the abovementioned risks.

Some authors proposed awake thoracic surgery under sole thoracic epidural or locoregional anaesthesia, avoiding general anaesthesia, with the purpose of maintaining a more physiologic cardiopulmonary and neurologic status [2,6-9]. However, some episodes of cough with unexpected lung movements were reported during the manipulation of the bronchial tree, hampering surgical movements and requiring further anaesthetic manoeuvres (e.g. vagal nerve blockade) with added risks [6-7]; panic attacks were described too, with the consequent need for urgent tracheal intubation [9].

Recently some case series and small studies were published about VATS with supraglottic airway devices, when spontaneous ventilation was permitted under deep sedation and lung isolation was achieved by means of the natural collapse of the lung due to iatrogenic pneumothorax created at the opening of the pleura [10-14]. These papers suggested 
that VATS could be managed safely and successfully with laryngeal mask in spontaneously breathing patients [10-13]. Excellent deflation of the lung and adequate surgical exposure were reported, without coughing during bronchial dissection. Moreover, only few episodes of moderate hypercapnia and minimal hypoxia were described, they we all easily treated by assisting the ventilation through the laryngeal mask and increasing the fraction of inspired oxygen in the fresh gas mixture. Compared with awake VATS, deep sedation improves patient satisfaction with less anxiety and no panic attack; compared with intubated VATS, the use of supraglottic airway devices minimizes the adverse effects of tracheal intubation, offering shorter anaesthetic induction time and better pain scores [13]. However, patient selection must be strict and intraoperative vigilance must be very high because of the risk for urgent conversion to an intubated technique with the patient in the challenging lateral position.

An alternative technique was described by Ozaki, et al. they used a laryngeal mask with a bronchial blocker to provide lung isolation in a patient difficult airway management, who was scheduled for biopsy of a pleural tumour [15]. Likewise, $\mathrm{Li}$, et al. successfully applied a similar approach in paediatric thoracoscopic anterior spinal release procedures for idiopathic scoliosis [16]. Subsequently the same group published a prospective pilot study of 55 patients undergoing thoracic surgery procedures with the need for one lung ventilation: 26 were managed with supraglottic airway device and endobronchial blocker and 29 with traditional dual lumen tube [17]. In this paper the laryngeal mask with the bronchial blocker was proved to allow for a satisfactory surgical exposure via one lung ventilation, and additionally was associated with less hemodynamic changes, fewer airway damages and reduced postoperative sore throat and hoarseness. Similar results were obtained in a prospective randomized study by Wang, et al. conducted on 100 adult patients scheduled for thoracoscopic procedures: between intubated patients with dual lumen tube and not-intubated patients with laryngeal mask and bronchial blocker there were no significant differences regarding the time for airway management and success rate of lung isolation; furthermore, arterial oxygen tension, end-expiration tidal volume, and peak airway pressure were comparable in the two groups [18]. More recently Sawasdiwipachai, et al. reported their experience with the use of the endobronchial blocker trough the supraglottic airway device in elective thoracic surgery [19]. Almost $97 \%$ of the patients were effectively managed with the described technique and the bronchial blocker was successfully placed during the first attempt in $86 \%$ of the patients. However, dislodgement of the endobronchial blocker occurred in $14 \%$ of the patients and displacement of the laryngeal mask occurred in $10 \%$ of the patients. The authors also reported that the incidence of sore throat (28\%) and hoarseness of voice $(17 \%)$ were higher than the previous studies. They concluded that for elective cases the use of a supraglottic device together with an endobronchial blocker is highly feasible when careful selection criteria are applied, but special attention and vigilance are required. Other case reports described a similar anaesthetic approach for thoracic procedures in patients with difficult or distorted airway [20-22] or tracheostomy [23].

In all the above mentioned works about supraglottic device with bronchial blocker, patients were curarized intraoperatively. Indeed, to our knowledge, our case series is the first report of the use of I-gel laryngeal mask with Arndt endobronchial blocker for thoracic surgery without neuromuscular blocking. Postoperative residual neuromuscular blockade is associated with impaired recovery, especially in thoracic surgery patients, who need to have effective cough minutes after surgery [3]. Although we did not administer any neuromuscular blocking drugs, we did not experience episodes of cough or unforeseen lung movements; neither bronchial blocker nor laryngeal mask dislodgment occurred.

An undoubted advantage of the combination of supraglottic device and bronchial blocker is the almost full control of airway: if tracheal intubation were required, a traditional dual lumen tube could be easily and quickly inserted, using the endobronchial blocker as a guide wire, even in urgent situation with the patient in lateral decubitus position.

The most critical concern about the application of laryngeal mask for thoracic procedure with one lung ventilation is the airway sealing: leakage of the supraglottic device occurs mainly when the patients are placed in the lateral position because of head and neck movements, and when higher airway pressure is needed to allow complete reexpansion of the collapsed lung after the surgery $[18,19]$. Indeed, some studies demonstrated that median seal pressure with laryngeal mask was around $30 \mathrm{cmH}_{2} \mathrm{O}$ with pressure above $40 \mathrm{~cm} \mathrm{H}_{2} \mathrm{O}$ in $20 \%$ of cases [24]. In our case series peak airway pressure never exceeded $32 \mathrm{cmH}_{2} \mathrm{O}$ and no significant air leakage was testified. Another worry about our anaesthesiologic technique is injury to the larynx and trachea, as result of long-term compression and shear by the supraglottic device and the blocker over the mucosa. In our experience, with procedures lasting about an hour no sore throat and hoarseness of voice were complained; however, some authors reported a higher incidence of throat ache, presumably due to a more prolonged duration of surgery [19]. Therefore, the combination of laryngeal mask and endobronchial blocker should be reserved for brief procedures.

\section{Conclusion}

In conclusion, the combination of the I-gel laryngeal mask and the Arndt endobronchial blocker without neuromuscular blockade can provide a feasible and alternative approach for lung isolation in thoracic surgery, particularly for short-term minimally invasive procedures.

Special vigilance is mandatory to constantly guarantee proper airway seal, and to avoid and to promptly detect laryngeal mask or bronchial blocker displacement.

Further larger-scale randomized comparative studies between this technique and conventional dual lumen intubation are needed to prove its safety.

\section{Acknowledgments}

None.

\section{Informed Consent}

Written informed consent was obtained from the patient for publication of this manuscript and any accompanying images.

\section{Declarations of interest}

None.

\section{Funding}

We declare no external financial support.

\section{References}

1. Minambres E, Buron J, Ballesteros MA, Llorca J, Muñoz P, et al. (2009) Tracheal rupture after endotracheal intubation: a literature systematic review. Eur J Cardiothorac Surg 35: 1056-1062. [Crossref]

2. Lohser J, Slinger P (2015) Lung injury after one-lung ventilation: A review of the pathophysiologic mechanisms affecting the ventilated and the collapsed lung. Anesth Analg 121: 302. [Crossref] 
3. Murphy GS, Szokol JW, Avram MJ, Greenberg SB, Shear T, et al. (2013) Postoperative residual neuromuscular blockade is associated with impaired clinical recovery. Anesth Analg 17: 133-141. [Crossref]

4. Zheng H, Hu XF, Jiang GN, Ding J-A, Zhu Y-M (2017) Nonintubated-awake anesthesia for uniportal video-assisted thoracic surgery procedures. Thorac Surg Clin 27: 399-406. [Crossref]

5. Whitehead T, Slutsky AS (2002) The pulmonary physician in critical care * 7: ventilator induced lung injury. Thorax 57: 635-642. [Crossref]

6. Chen KC, Cheng YJ, Hung MH, Tseng Y-D, Chen J-S (2012) Nonintubated thoracoscopic lung resection: a 3-year experience with 285 cases in a single institution. J Thorac Dis 4: 347-351. [Crossref]

7. Guo Z, Shao W, Yin W, Chen H, Zhang X, et al. (2014) Analysis of feasibility and safety of complete video-assisted thoracoscopic resection of anatomic pulmonary segments under non-intubated anesthesia. J Thorac Dis 6: 37-44. [Crossref]

8. Liu J, Cui F, Li S, Chen H, Shao W, et al. (2015) Nonintubated video-assisted thoracoscopic surgery under epidural anesthesia compared with conventional anesthetic option: A randomized control study. Surg Innov 22: 123-130. [Crossref]

9. Mineo TC, Tamburrini A, Perroni G, Ambrogi V (2016) 1000 cases of tubeless videoassisted thoracic surgery at the Rome Tor Vergata University. Future Oncol 12: 13-18. [Crossref]

10. Ambrogi MC, Fanucchi O, Gemignani R, Guarracino F, Mussi A (2012) Video assisted thoracoscopic surgery with spontaneous breathing laryngeal mask anesthesia: preliminary experience. J Thorac Cardiovasc Surg 144: 514-515.

11. Ambrogi MC, Fanucchi O, Korasidis S, Davini F, Gemignani R, et al. (2014) Nonintubated thoracoscopic pulmonary nodule resection under spontaneous breathing anesthesia with laryngeal mask. Innovations (Phila) 9: 276-280. [Crossref]

12. Gonzalez-Rivas D, Fernandez R, de la Torre M, Rodriguez JL, Fontan L, et al. (2014) Single-port thoracoscopic lobectomy in a nonintubated patient: The least invasive procedure for major lung resection? Interact CardioVasc Thorac Surg 19: 552-555. [Crossref]

13. Irons JF, Miles LF, Joshi KR, Klein AA, Scarci M, et al. (2017) Intubated versus nonintubated general anesthesia or video-assisted thoracoscopic surgery. A CaseControl Study. J Cardiothorac Vasc Anesth 31: 411-417. [Crossref]
14. Zhang RX, Li Y, Liu XB, Lu X-H, Sun H-B, et al. (2018) Is laryngeal mask airway general anesthesia feasible for minimally invasive esophagectomy? J Thorac Dis 10: E210-E213. [Crossref]

15. Ozaki M, Murashima K, Fukutome T (2004) One-lung ventilation using the ProSeal ${ }^{\mathrm{TM}}$ laryngeal mask airway. Anaesthesia 59: 719-729.

16. Li P, Liang W, Gu H (2009) One-lung ventilation using ProSeal ${ }^{\mathrm{TM}}$ laryngeal mask airway and Arndt endobronchial blocker in paediatric scoliosis surgery. $\mathrm{Br} J$ Anaesth 103: 902-903. [Crossref]

17. Li Q, Li P, Xu J, Gu H, Ma Q, et al. (2014) A novel combination of the Arnd endobronchial blocker and the laryngeal mask airway ProSeal ${ }^{\mathrm{TM}}$ provides one-lung ventilation for thoracic surgery. Exp Ther Med 8: 1628-1632. [Crossref]

18. Wang S, Zhang J, Cheng H, Yin J, Liu X (2014) A clinical evaluation of the ProSeal laryngeal mask airway with a Coopdech bronchial blocker for one-lung ventilation in adults. J Cardiothorac Vasc Anesth 28: 900-903.

19. Sawasdiwipachai P, Boonsri S, Suksompong S, Prowpan P (2015) The uses of laryngea mask airway ProSeal ${ }^{\mathrm{TM}}$ and endobronchial blocker for one lung anesthesia. $J$ Anesth 29: 660-665.

20. Tsuchihashi T, Ide S, Nakagawa H, Hishinuma N, Takano T, et al. (2007) Differential lung ventilation using laryngeal mask airway and a bronchial blocker tube for a patient with unanticipated difficult intubation. Masui 9: 1075-1077. [Crossref]

21. Wexler S, Ng JM (2010) Use of the ProSeal laryngeal mask airway and Arndt bronchial blocker for lung separation in a patient with a tracheal mass and aspiration risk. $J$ Cardiothorac Vasc Anesth 24: 215-216.

22. Rispoli M, Nespoli MR, Viscardi D, Zani G, Bizzarri F, et al. (2017) One lung ventilation with laryngeal mask ProSeal ${ }^{\mathrm{TM}}$ and $\mathrm{EZ}-$ blocker $^{\mathrm{TM}}$ in a partial laryngectomized patient. J Clin Anesth 38: 57-58.

23. Robinson AR, Gravenstein N, Alomar-Melero E, Peng YG (2008) Lung isolation using a laryngeal mask airway and a bronchial blocker in a patient with a recent tracheostomy. $J$ Cardiothorac Vasc Anesth 22: 883-886. [Crossref]

24. Cook TM, Lee G, Nolan JP (2005) The ProSeal laryngeal mask airway: A review of the literature. Can J Anesth 52: 739-760. [Crossref]

Copyright: $\odot 2020$ Di Gregorio G. This is an open-access article distributed under the terms of the Creative Commons Attribution License, which permits unrestricted use, distribution, and reproduction in any medium, provided the original author and source are credited. 